\title{
Performance and Analysis of CDM-FH-OFDMA for Broadband Wireless Systems
}

\author{
Kan Zheng, Lu Han, Jianfeng Wang, and Wenbo Wang \\ Wireless Signal Processing Lab, \\ Beijing University of Posts \& Telecomms, Beijing, China \\ zkan@buptnet.edu.cn
}

\begin{abstract}
Frequency-hopping (FH) methods in the Orthogonal frequency division multiplexing access(OFDMA) system, which are to assign user-specific subcarrier to the active users, have been paid much attention to in the broadband wireless communication system. In this paper, we present a novel multiple access scheme, referred to as CDMFH-OFDMA, which is the extension of FH-OFDMA with code division multiplexing (CDM). This scheme can exploit the frequency diversity gain without the aid of channel coding. And it also can be employed in the multi-cell environment with one frequency reuse factor. Computer simulation demonstrates effectiveness of CDM-FH-OFDMA and the conclusion is followed.
\end{abstract}

Keywords: OFDM, FH, CDM.

\section{Introduction}

Orthogonal frequency division multiplexing (OFDM) as a modulation technique is being applied extensively to future wireless broadband systems due to its efficient usage of the available frequency bandwidth and robustness to frequency selective fading environments[1]2. Meanwhile, code division multiple access (CDMA) has already shown quite a bit of promise in its spectral efficiency through the flexible frequency reuse and multiple access technique for cellular systems $[3$. So OFDM combined with code division multiple access (CDMA) has drawn a lot of interests in the research of future mobile communication systems 4 [5. These OFDM-CDMA schemes are derived from the classic DSCDMA approach, employing mutually orthogonal spreading codes for user separation within one cell, and scrambling codes for distinguishing different cells.

An alternative to using CDMA for multiple-access is to assign user-specific subcarrier to the active users. These schemes are known as OFDMA or frequency-hopping OFDMA 2]. These schemes maintain the user orthogonality also on frequency-selective fading channels, but rely solely on channel coding and interleaving for obtaining the diversity gain. However, it is claimed that these scheme will be more sensitive to inter-cell-interference and therefore not suitable in frequency-reuse-one systems $\underline{6}$. 
To find out the most appropriate multiple access schemes, it is necessary to investigate the performances of different multiple access schemes not only in the one-cell scenarios but also in the multi-cell scenarios.

In this paper, we propose a novel multiple access scheme, referred to as CDM-FH-OFDMA, which is the extension of FH-OFDMA with code division multiplexing (CDM). Similar to FH-OFDMA, it applies OFDMA for user separation but additionally uses CDM on the data symbols belonging to the same user. The CDM component is introduced in order to achieve additional frequency diversity gain and average the the inter-cell-interference. Like OFDMCDMA, this CDM-FH-OFDMA exploits the advantages given by the combination of the spread spectrum technique and multi-carrier modulation. Since one user exclusively uses each subset of subcarriers, there is no multiple access interference between different users in the same cell. And the self-interference of one user can be easily decreased by interference cancellation since all superimposed modulated spreading codes of its subcarrier subset are affected by the same channel fading. When considering the cellular system, frequency reuse factor of one can be realized by using different scramble codes in the neighbor cells and inter-cell interference can be avoid by selecting different subcarrier set for the users in the neighboring cells if the system load is not heavy.

This paper is organized as follows. Section 2 gives the brief description of CDM-FH-OFDMA system. The detector structure is described in Section 3. Section 4 describes the simulation configurations, and in Section 5 the simulation results are presented and discussed. Finally, Section 6 gives the conclusion.

Notations: Throughout this paper, matrices and vectors are set in boldface. ()$^{T},()^{*}$ and ()$^{+}$denote transpose, conjugate transpose and Moore-Penrose pseudo-inverse, respectively.

\section{System Model}

Fig 1 shows the block diagram of a CDM-FH-OFDMA system. As the requirement of frequency-hopping method, each frame, which the basic process unit in the system, consists of the $N_{t}$ OFDM symbols.After the information bits of

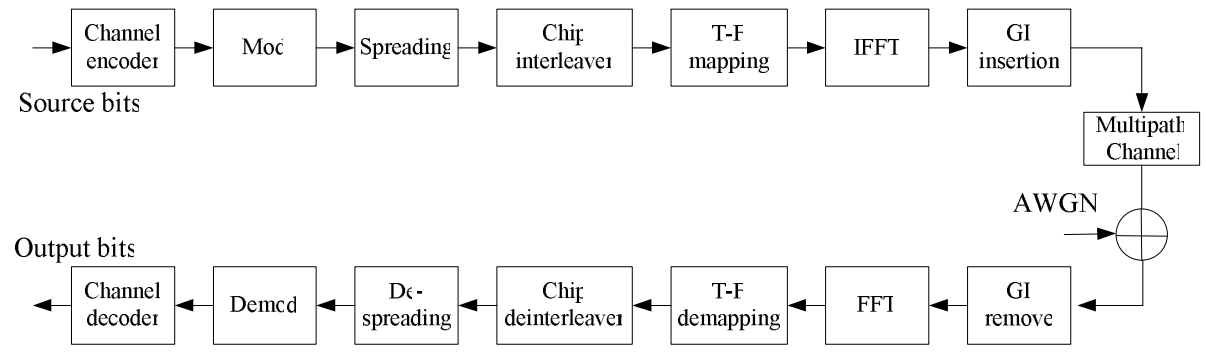

Fig. 1. Block diagram of CDM-FH-OFDMA system 
user $m$ are encoded and interleaved, they are modulated to the complex-value symbols with the rate of $1 / T_{d}$. The vector

$$
\mathbf{d}_{i}^{(m)}=\left[\begin{array}{llll}
d_{i, 0}^{(m)} & d_{i, 1}^{(m)} & \cdots & d_{i, Q-1}^{(m)}
\end{array}\right]^{T}
$$

represents one block of $Q$ parallel modulated data symbols of user $m$ in the $i$ th OFDM symbol.Each data symbol is multiplexed with another orthogonal spreading code of length $L$. The $L \times Q$ matrix

$$
\mathbf{C}=\left[\begin{array}{lll}
\mathbf{c}_{0} & \mathbf{c}_{1} \cdots \mathbf{c}_{Q-1}
\end{array}\right]
$$

represents the $Q$ different spreading codes $\mathbf{c}_{q}=\left[\begin{array}{ll}c_{q, 0} & c_{q, 1} \cdots c_{q, L-1}\end{array}\right]^{T} \in \mathbb{C}^{L \times 1}, 0 \leq$ $q \leq Q-1$, used by user $m$, which are the combination of the orthogonal Walsh codes and the base-station-specific scrambling codes. The spreading matrix $\mathbf{C}$ can be same for all the users. The modulated spreading signals are synchronously added, resulting in the transmission vector in the $i$ th OFDM symbol.

$$
\mathbf{S}_{i}^{(m)}=\mathbf{C d}_{i}^{(m)}=\left[S_{0}^{(m)} S_{1}^{(m)} \cdots S_{L-1}^{(m)}\right]^{T} \in \mathbb{C}^{L \times 1}
$$

To obtain OFDMA scheme, the orthogonal spreading matrix is replaced by the identity matrix.

As shown in Fig 2, in a CDM-FH-OFDMA system, each user may be assigned a specific frequency-hopping sequence that indicates the specific subcarrier subset to use for data transmission in each OFDM block interval,i.e. specific TimeFrequency(T-F) mapping pattern. Multiple data transmissions for multiple users

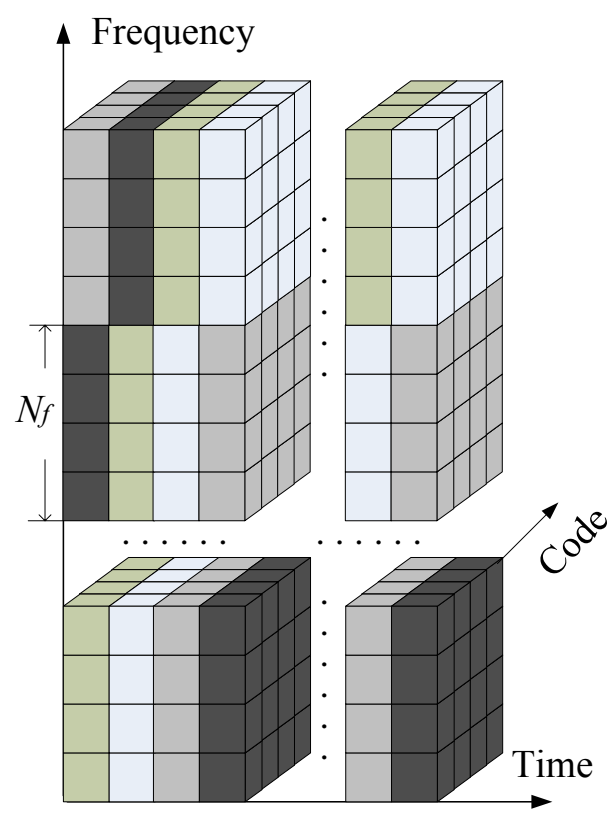

Fig. 2. Multiple access method of CDM-FH-OFDMA system 
may be sent simultaneously using different frequency-hopping sequences that are orthogonal to one another, so that only one data transmission uses each subcarrier subset in each OFDM block interval. Using orthogonal frequency-hopping sequences, the multiple data transmissions do not interfere with one another while enjoying the benefits of frequency diversity. For brevity, but without loss of generality, the size of subcarrier subset $N_{f}$ is assumed to be same as the length of the spreading code,i.e. $N_{f}=L$. Then, according to the frequency-hopping sequence of user $m$, the spreading signal of user $m$ is modulated by IDFT onto different subsets of $L$ subcarriers within the $N$ total available subcarriers in one frame ,and the resultant signal in the $i$ th OFDM block interval can be expressed as

$$
s_{i}^{(m)}(n)=\frac{1}{\sqrt{N}} \sum_{l=0}^{L-1} S_{l}^{(m)} e^{j 2 \pi\left(l+P_{i}^{(m)} L\right) n / N}
$$

where $P_{i}^{(m)}$ is a hopping index which indicates subcarrier subset indices for the $i$ th OFDM symbol of user $m$ and the T-F mapping pattern for user $m$ is

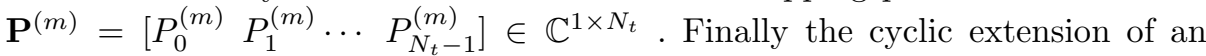
OFDM block is added as guard interval before transmission in order to avoid the inter-symbol-interference.

The transmitted signals of different users propagate through independent frequency-selective fading channels. And the channel is modelled as a wide sense stationary, uncorrelated scattering (WSSUS), Rayleigh fading channel with $L_{t}$ paths and it is assumed that the channel state remains unchanged during at least one OFDM block. Then the channel impulse response during the $i$ th OFDM symbol can be expressed as

$$
h^{(m)}(i ; n)=\sum_{l=0}^{L_{t}-1} \alpha_{l}^{(m)}(i) \delta\left(n-\tau_{l}^{(m)}\right)
$$

where the $l$ th tap gain $\alpha_{l}^{(m)}(i)$ with propagation delay $\tau_{l}^{(m)}$ of the $m$ th user is independent complex Gaussian random variance with zero mean and variable of $\sigma_{m, l}^{2}$.If the cyclic prefix accommodates the channel delay spread between base station and terminals, it is assumed that the narrowband signal that is transmitted through each subcarrier experiences flat Rayleigh fading channel. The flat fading coefficient at the $k$ th subcarrier of the $m$ th user during the $i$ th OFDM block interval can be expressed as

$$
\begin{gathered}
H_{i, k}^{(m)}=\sum_{l=0}^{L_{t}-1} \alpha_{l}^{(m)} e^{-j 2 \pi \tau_{l}^{(m)} k / N}, \\
0 \leq i \leq N_{t}-1,0 \leq k \leq N-1
\end{gathered}
$$

After cyclic prefix removal and DFT, the received signals of the desired users can be easily separated according to the hopping pattern and no interference between users exists under the assumption of ideal synchronization since the 
users are distinguished by an FDMA scheme. The signal vector of user $m$ in the $i$ th OFDM symbol can be written as

$$
\mathbf{Y}_{i}^{(m)}=\mathbf{H}_{i}^{(m)} \mathbf{S}_{i}^{(m)}+\mathbf{W}_{i}=\left[Y_{i, P_{i}^{(m)} L}^{(m)} Y_{i, P_{i}^{(m)} L+1}^{(m)} \cdots Y_{i,\left(P_{i}^{(m)}+1\right) L-1}^{(m)}\right]^{T} \in \mathbb{C}^{L \times 1}(7)
$$

where the $L \times L$ channel fading diagonal matrix for the desired user, AWGN term with zero mean and variance of $\sigma_{n}^{2}$ are given respectively by

$$
\begin{aligned}
& \mathbf{H}_{i}^{(m)}=\operatorname{diag}\left\{H_{i, P_{i}^{(m)} L}^{(m)}, H_{i, P_{i}^{(m)} L+1}^{(m)}, \cdots, H_{i,\left(P_{i}^{(m)}+1\right) L-1}^{(m)}\right\} \\
& \mathbf{W}_{i}=\left[\begin{array}{ll}
W_{i, 0} & W_{i, 1} \cdots W_{i, L-1}
\end{array}\right]^{T} \in \mathbb{C}^{L \times 1}
\end{aligned}
$$

\section{Detector Structure}

Any of the single-user or multi-user detection techniques presented for MCCDMA systems 4 can be applied for the detection of the data of a single user in CDM-FH-OFDMA systems.

Firstly the received signal vector is equalized by employing a bank of adaptive one-tap equalizers to combat the phase and amplitude distortions caused by the mobile fading channel on the subcarriers. The one-tap equalizer is simply realized by one complex-valued multiplication per subcarrier. The received sequence at the output of the equalizer in the $i$ th OFDM block interval can be expressed as

$$
\mathbf{Z}_{i}^{(m)}=\mathbf{G}_{i}^{(m)} \mathbf{Y}_{i}^{(m)}
$$

The diagonal equalizer matrix

$$
\mathbf{G}_{i}^{(m)}=\operatorname{diag}\left\{G_{i, 0}^{(m)}, G_{i, 1}^{(m)}, \cdots, G_{i, L-1}^{(m)}\right\} \in \mathbb{C}^{L \times L}
$$

represents the $L$ complex-valued equalizer coefficients of the subcarriers assigned to $S_{i}^{(m)}$. The complex-valued output $\mathbf{Z}_{i}^{(m)}$ is despread by correlating it with the spreading matrix $\mathbf{C}$. The complex-valued soft-decided values at the output of the despreader is

$$
\tilde{\mathbf{d}}_{i}^{(m)}=\mathbf{C}^{T} \mathbf{Z}_{i}^{(m)}=\left[\begin{array}{llll}
\tilde{d}_{i, 0}^{(m)} & \tilde{d}_{i, 1}^{(m)} \cdots & \tilde{d}_{i, Q-1}^{(m)}
\end{array}\right]^{T} \in \mathbb{C}^{Q \times 1}
$$

The data symbol in the hard-decided detected vector $\hat{\mathbf{d}}_{i}^{(m)}=\left[\hat{d}_{i, 0}^{(m)} \hat{d}_{i, 1}^{(m)} \ldots\right.$ $\left.\hat{d}_{i, Q-1}^{(m)}\right]^{T} \in \mathbb{C}^{Q \times 1}$ is given by

$$
\hat{d}_{i, q}^{(m)}=\Gamma\left\{\tilde{d}_{i, q}^{(m)}\right\}, 0 \leq q \leq Q-1
$$

where $\Gamma\{*\}$ is the quantization operation according to the chosen data symbol alphabet.

Several different diversity combining techniques have been proposed in the literature. In this paper the equalizer according to the minimum mean error 
square(MMSE) is used in the despreading by choosing the equalization coefficients as

$$
G_{i, l}^{(m)}=\frac{H_{i, P_{i}^{(m)} L+l}^{(m) *}}{Q\left|H_{i, P_{i}^{(m)} L+l}^{(m)}\right|^{2}+\sigma_{n}^{2}}, 0 \leq l \leq L-1
$$

The detector described above is originated from the principle of single-user detection (SD), which only detects the desired signal without taking into account any information about multi-code interference. With the number of spreading vectors (i.e. $Q$ ) is increased, the performance of such SD detector will be deteriorated due to more serious multi-code interference. The interference cancellation (IC) can improve the performance of the system with heavy load at the expense of higher receiver complexity. The principle of parallel interference cancellation (PIC) is introduced [7] and can also be applied to CDM-FHOFDMA systems. The basic idea is to take the estimated data obtained by the initial SD detector and regenerate the transmitted signals to calculate the occurring multi-code interference of each desired data signal caused by all other spreading signals, then to subtract it from the received signals. When only the $q$ th, $0 \leq q \leq Q-1$,transmitted data symbol $d_{q}^{(m)}$ of user $m$ is desired and others are regarded as interference,the corresponding signal after interference-reduced can be written as

$$
\mathbf{Y}_{i, q}^{(m)}=\mathbf{Y}_{i}^{(m)}-\mathbf{H}_{i}^{(m)} \mathbf{C}_{q}^{-} \mathbf{d}
$$

where $\mathbf{C}_{q}^{-}$denotes the modified spreading matrix obtained by zeroing the $q$ th column of $\mathbf{C}$.

After this cancellation step, the next data detection including equalization

and despreading/combining are applied to the interference-reduced signal $\mathbf{Y}_{i, q}^{(m)}$, leading to more reliable data estimations than the initial SD detection. The equalization coefficients after interference-reduction are changed to

$$
G_{i, l}^{(m)}=\frac{H_{i, P_{i}^{(m)} L+l}^{(m) *}}{\left|H_{i, P_{i}^{(m)} L+l}^{(m)}\right|^{2}+\sigma_{n}^{2}}, 0 \leq l \leq L-1
$$

In each stage, $Q$ interference cancellation and data estimation will be performed. This iterative procedure can be continued until the performance is satisfied.

\section{System Configuration}

The key simulation parameters are summarized in Table 1 and are kept in same with [2] in order to be compatible with FH-OFDMA system, which has been widely studied in $3 \mathrm{GPP}$ long-time evolution. The selective fading channel models including PB3, VA120 defined by ITU are used in the simulations 9. Each path of the channel is modelled as a classical Jakes Doppler spectrum. Under the assumption of a quasi-stationary channel, the channel is constant during one OFDM interval. At the receiver, perfect symbol/carrier synchronization and channel state information are assumed to be available. 
Table 1. System Parameters

\begin{tabular}{ll}
\hline Carrier & $2 \mathrm{GHz}$ \\
\hline Frame duration $(m s)$ & 2 \\
\hline DFT size & 1024 \\
\hline Cyclic Prefix interval (samples/ $\mu s)$ & $64 / 9.803$ \\
\hline Subcarrier separation $(\mathrm{kHz})$ & 6.375 \\
\hline OFDM block duration $(\mu s)$ & 166.67 \\
\hline Number of OFDM symbols per frame $N_{t} 12$ \\
\hline Number of useful data subcarriers & 600 \\
\hline Size of data subcarrier subset & 40 \\
\hline Number of spreading codes & 8 \\
\hline Channel coding/Decoding & Turbo codec/ \\
& MAX_LOG_MAP \\
\hline Channel model & (iteration=8) \\
\hline Spreading factor $(\mathrm{Q})$ & PB3, VA120 $[9]$ \\
\hline Modulation & 8 \\
\hline
\end{tabular}

Table 2. Information bit payload and code block sizes for transport format

\begin{tabular}{|c|c|c|c|c|c|c|}
\hline Modul & Code & Informatic & 24-bit-C & Code Block & $\overline{\mathrm{R}}=1 / 3(\mathrm{~K}=4)$ & Rate \\
\hline & Rate & bit payload & addition & Segmentation & Turbo-coding & matching \\
\hline$\overline{\text { QPSK }}$ & $1 / 2$ & 480 & 504 & $1 \times 504$ & 1524 & 960 \\
\hline QPSK & $2 / 3$ & 640 & 664 & $1 \times 664$ & 2004 & 960 \\
\hline QPSK & $4 / 5$ & 768 & 792 & $1 \times 792$ & 2388 & 960 \\
\hline
\end{tabular}

\subsection{Interleaving}

The interleavers have to be applied in the CDM-FH-OFDMA systems in order to better explore the diversity gain inherent in the time-frequency selective fading channel. There are two positions that the interleaving will be performed at the transmitter. One is before modulation module, i.e. bit-interleaving, the other is before the IDFT,i.e. chip-interleaving. Since all the data symbols are processed according to the frame unit in the time domain, the interleaving will be applied within one frame including $N_{t}$ OFDM symbols.

In the transmitter, the binary information is first coded with CRC attachment and code block segmentation before channel coding. The coded bit is punctured and bit-interleaved according to [8]. Table 2 provides appropriate information bit payload and code block segmentation values for the test cases in the simulations. For the sake of implication,only one transport block size for each user is assumed in this paper. Other sizes may also be evaluated if necessary.

A block interleaver is applied in case of chip-interleaving, which is a matrix with $N_{\text {depth }}$ rows and $N_{\text {length }}$ columns. The symbols are written into the matrix by rows and read out afterward by columns. The deinterleaver puts the symbols into a matrix with the same size, but the symbols are written by columns 
and read out by rows. Usually the number of rows $N_{\text {depth }}$ is defined as the interleaving depth while the number of the column $N_{\text {length }}$ as the interleaving length. In the simulations, the $N_{\text {depth }}$ and $N_{\text {length }}$ of the block chip-interleaving equal to the size of the subcarrier subset and the number of OFDM symbol per frame,respectively.

\subsection{Frequency-Hopping Pattern and Spreading}

The different users are distinguished by allocating each user with a separate pattern for the time-frequency(T-F) mapping of OFDM units. All the T-F mapping patterns in a cell should be orthogonal to avoid the cross-interference between different users.Each of them should provide not only a maximized diversity gain within a cell but also a minimized inter-cell-interference between the neighboring cells $[2$.

Therefore,the set of 15 orthogonal T-F pattern, one for each user, is derived from a single generic Costas sequence of length 15 . Since the number of OFDM symbol per frame is 12 (i.e. $N_{t}=12$ ), the right T-F pattern of length $N_{t}$ is obtained by discarding the last three symbols of the generic Costas sequence. Then, the first T-F mapping pattern is given by

$$
\mathbf{P}^{(0)}=\left[\begin{array}{llllll}
13 & 53921411 & 15412710
\end{array}\right]
$$

All the T-F pattern in the set are obtained from the first patten in the set by all the different cyclic shifts in the frequency domain. In that way, it is ensured that the set of pattern is orthogonal.

All the time-frequency mapping patterns in a frame are cyclically time-shifted by a cell-specific offset, corresponding to an integer number of OFDM symbols. The time offset is changed for each frame, according to a cell-specific scrambling sequence. In that way, even if the two cells are synchronous in one frame, they will very probably be asynchronous in the next frame, resulting in a minimized cross-interference, as predicted by the correlation properties of time-frequency mapping patterns.

In the simulated CDM-FH-OFDMA system, the data subcarrier subset size $N_{f}$ is larger than the spreading code length $L$. So there are $N_{f} / L$ spreading signal of length $L$ within one data subcarrier subset of size $N_{f}$.

\section{Performance Evaluation}

\section{$5.1 \quad$ Single-Cell}

In Fig 3(a) and Fig 3(b), the performances of the proposed CDM-FH-OFDMA and FH-OFDMA system applying MMSE principle with the different code rates under PB3 channel or VA120 channel are compared, where QPSK modulation is used. In case of low or medium code rate, the BLER performances of FHOFDMA are equivalent to or better than these of CDM-FH-OFDMA because it can well exploit the frequency diversity gain through channel coding and 


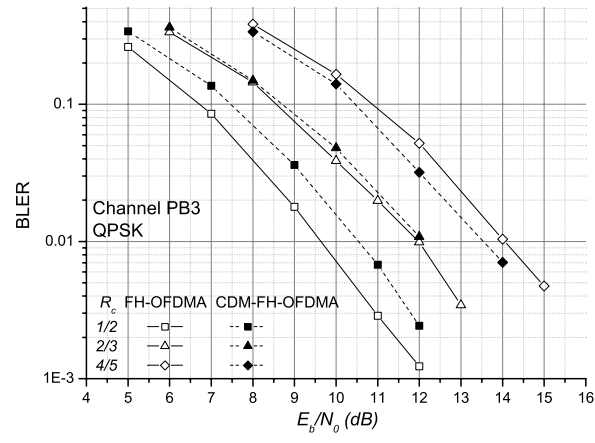

(a) PB3

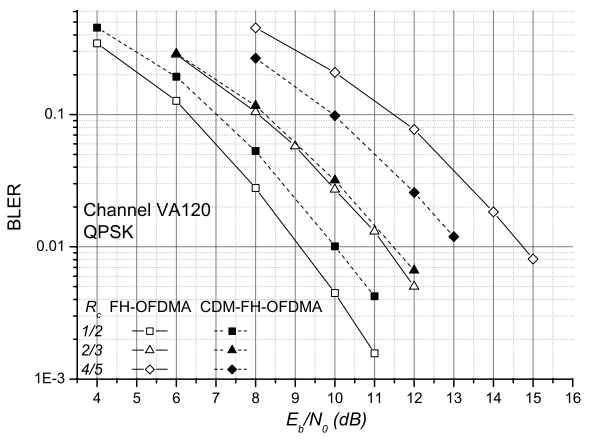

(b) VA120

Fig. 3. BLER performances in FH-OFDMA or CDM-FH-OFDMA using MMSE detector

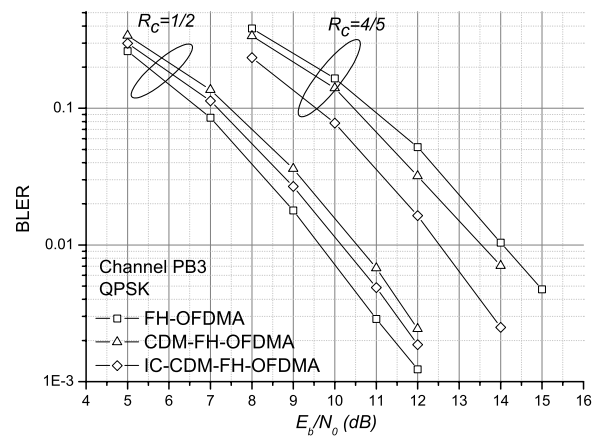

(a) PB3

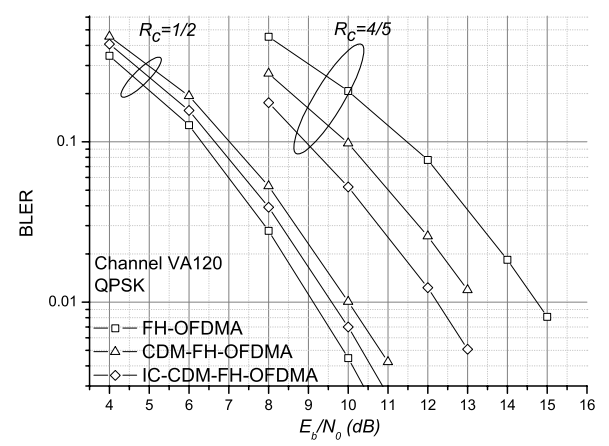

(b) VA120

Fig. 4. BLER performances in FH-OFDMA or CDM-FH-OFDMA using PIC detector

the multi-code interference in CDM-FH-OFDMA deteriorates the BLER performance. However, with the high code rate, the CDM-FH-OFDMA system can make better use of the frequency diversity gain by the operations of spreading and combination than the channel coding. For example, if the target BLER is assumed to be $10^{-2}$, the BER gain of CDM-FH-OFDMA compared with FHOFDMA is about 1 or $2 \mathrm{~dB}$ under PB3 channel or VA120 channel in case of $R_{c}=4 / 5$.

Fig 4(a) and Fig 4(b) and compare the performances of the proposed CDMFH-OFDMA and FH-OFDMA system applying MMSE principle or PIC detector with the different code rates under PB3 channel or VA120 channel, where QPSK modulation is used. When the code rate is low (e.g. $\left.R_{c}=1 / 2\right)$, the performance of CDM-FH-OFDMA system are mainly affected by the channel coding/decoding and the interference cancellation won't achieve much performance gain. Then, the BLER performance of CDM-FH-OFDMA system with PIC still keeps little worse than that of FH-OFDMA system. On the other hand, with the higher 
code rate(e.g. $\left.R_{c}=4 / 5\right)$, the $\mathrm{PIC}$ detector will give about $1 \mathrm{~dB}$ gain if the target BLER is assumed to be $10^{-2}$ under both channel environments.

\section{$5.2 \quad$ Multi-cell}

One of the advantages of CDM-FH-OFDMA system is that the same bandwidth can be reused in each cell, which is often refereed to a full frequency re-use, or frequency re-use factor of 1 . The main benefit of such a frequency reuse is mainly ease of deployment, given that no frequency planning is required. However, the CDM-FH-OFDMA system with a frequency reuse of 1 becomes interferencelimited,and the interference perceived by the terminal from the different cells might not be perfectly white. First, the interfering signals undergo time dispersion, and hence, do not have a flat spectrum. Furthermore, if the OFDM units are not all being used in the interfering cells, the resulting spectrum from each of these partially-load interfering cells will contain gaps. It is therefore likely that the total interference spectrum observed by the terminal would not be flat, and hence, it might not be accurately modelled using white noise.

The impact of realistic inter-cell interference on performance has therefore been evaluated using the multi-cell simulator with a radio geometry concept, which consider relative inter-cell interference and independent fading for a limited number of chip-exact modelled terminal in a neighboring cell[10]. To become independent from absolute path gains as well as cell layouts, we use the geometry factor which is defined as

$$
G=\frac{I_{o r}}{I_{o c, b}+I_{o c}}
$$

where $I_{o r}$ denotes the received total power originated from the serving, $I_{o c}$ is the received total power from the all the interfering cells and $I_{o c, b}$ is the portion of the received power from those cells modelled as AWGN. Considering the tradeoff between the reliability and simulation complexity, the inter-cell-interference is assumed to be generated by a single neighboring cell. It is generated by using the same time-frequency mapping patterns as in the serving cell. Namely, in all cells, the time-frequency mapping patterns in a frame are cyclically time-shifted by a cell-specific offset, corresponding to an integer number of OFDM symbols. The time offset is changed for each frame, according to a cell-specific scrambling sequence.

For the sake of simplification, there is only one user in the serving cell and the inter-cell interference dominates over the noise in this simulation scenario. It is assumed that $100 \%$ of the additive interference plus noise is due to the inter-cell-interference and $0 \%$ due to the thermal noise, i.e.,

$$
\frac{I_{o c}}{I_{o c, b}+I_{o c}}=1
$$

The simulation results with the different cell load in the interfering cell are plotted in Fig 5, which gives the performances of FH-OFDMA and CDM-FHOFDMA with MMSE detection. Less geometry factor, further distance to the 


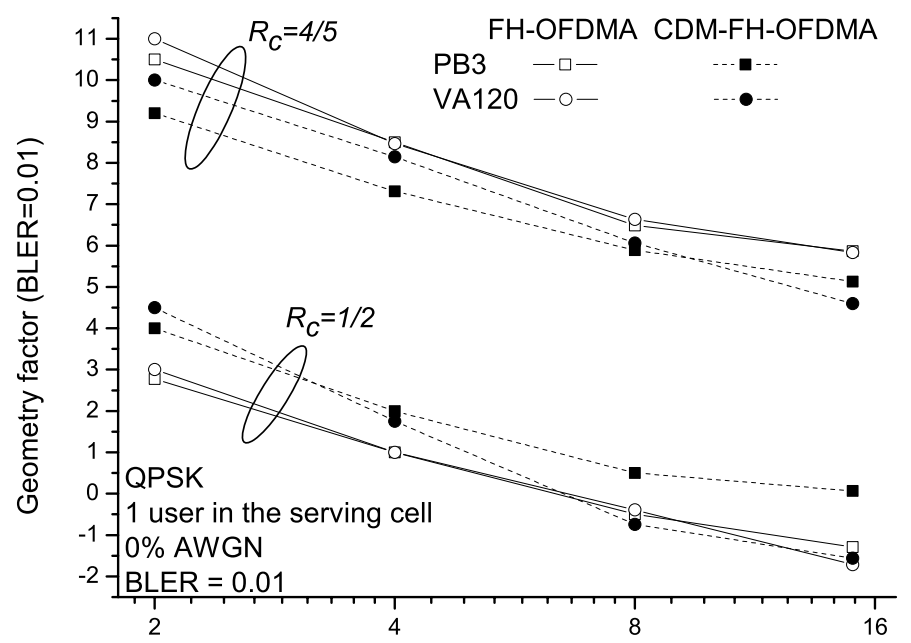

Fig. 5. Required geometry factor with $B L E R=10^{-2}$ under different cell load in the interfering cells

base station the user is. When the cell load in the interfering cell increases, the link-performance improves. This perhaps somewhat counterintuitive result is explained as follows. When the users in the interfering cells increases, the collision probability of the subcarriers between the serving user and the interfering users becomes larger. So the interference becomes more like Gaussian according to the center-limited principle. Meanwhile, the signal power of each interfering users decreased with the number of users increases in order to keep the total interference power constant. Therefore, the required geometry factor with $\mathrm{BLER}=10^{-2}$ decreased with the number of the users in the interfering cell,which means the serving user can get the same BLER performance in the further position to the centering base station.

In case of low code rate, the performances of FH-OFDMA are little better than those of CDM-FH-OFDMA because the channel coding can exploit the diversity gain well,which is similar to the single-cell case. However, with the code rate increases, the proposed CDM-FH-OFDMA system with the simple MMSE detection outperforms FH-OFDMA system.

\section{Conclusion}

In this paper,we propose a CDM-FH-OFDMA scheme for the downlink high data rate transmission in the broadband wireless communication system. Simulation results demonstrate that the performances of CDM-FH-OFDMA system are better than those of conventional FH-OFDMA in case of medium or high code rate. Also, the interference cancellation can be applied in CDM-FH-OFDMA systems to further improve the performance. 


\section{References}

[1] Eklund, C.; Marks, R.B.; Stanwood, K.L.; Wang, S,"IEEE standard 802.16: a technical overview of the WirelessMAN air interface for broadband wireless access ,"IEEE Communications Magazine,vol.40,no.6,pp.98-107,2002

[2] 3GPP TR 25.892. V2.0.0. (2004-06).Feasibility Study for OFDM for UTRAN enhancement. (Release 6)

[3] H. Holma and A. Toskala, WCDMA for UMTS. New York: Wiley, 2000.

[4] S .Hara,R .Prasad, "Overview of multicarrier CDMA," IEEE Communications Magazine,vol.35,no.12,pp.126 - 133,Dec. 1997

[5] K. Zheng,G. Zeng,W .Wang,"Performance Analysis for OFDM-CDMA with Joint Frequency-time Spreading," IEEE Transactions on Broadcasting, vol.51, no.1, pp.144- 148,March 2005

[6] Maeda, N.; Atarashi, H.; Abeta, S.; Sawahashi, M.;"Throughput comparison between VSF-OFCDM and OFDM considering effect of sectorization in forward link broadband packet wireless access," in Proc.IEEE Vehicular Technology Conference, vol.1, pp.47-51,Fall. 2002

[7] M.K.Varanasi, B.Aazhang, "Multistage detection in asynchronous code-division multiple-access communications," IEEE Trans. Commun.,vol.38,no.4,pp. 509 519, April 1990

[8] 3rd Generation Partnership Project (3GPP), 3G TS 25.211, v3.5.0, Physical Channels and Mapping of Transport Channels onto Physical Channels (FDD), Dec. 2000 .

[9] Guidelines for Evaluation of Radio Transmission Technologies for IMT-2000, Rec. ITU-R.M1225.

[10] Weber, R.; Schulist, M.; Schotten, H."WCDMA multi-cell link-level performance," Proc.IEEE Personal, Indoor and Mobile Radio Communications, vol.3,pp.1362 - 1366,Sept. 2002 